Swiss Journal of Integrative Medicine

\title{
Aus der Praxis für die Praxis
}

Schweiz Z Ganzheitsmed 2011;23:202-203

DOI: $10.1159 / 000329928$

\section{Zaubern in der Zahnarztpraxis}

\section{Mit Zauberkunststücken die Angst aus dem Behandlungszimmer pusten}

Wir alle, Fachleute und Patientinnen und Patienten, wissen heute, wie wichtig die Pflege unserer Zähne und eine gute Mundhygiene für unser gesamtes Wohlbefinden und die Gesundheit sind - und das unser ganzes Leben lang. Für die Erhaltung der Zähne sind darüber hinaus Zahnarztbesuche unerlässlich und von entscheidender Bedeutung.

Dennoch erleben Zahnärztinnen und Zahnärzte trotz allem Bemühen von Eltern und Mitarbeitern Kinder, die ängstlich, verschüchtert oder «bockig» sind. Sie verweigern sich total oder lassen die Untersuchung nur unter höchster Anspannung über sich ergehen. Oder sie haben kleine «Zappelphilippe», die offenbar nicht stillsitzen und mitarbeiten können. Und auch so mancher erwachsene Patient hat so sehr Angst, dass es viel Zeit und Kraft kostet, positiven Kontakt aufzubauen und die Behandlung entspannt und konzentriert durchzuführen.

Sachliche Informationen und vernünftige Argumente helfen dann meist zum Verzweifeln wenig. «Zaubern müsste man können!», mögen sich die Mitarbeiter in Zahnarztpraxen da manchmal denken. - Ja, warum eigentlich nicht? Zaubern Sie doch einfach wirklich! Denn ein verzauberter Patient ist ein entspannter Patient, einer, der glücklich und beschwingt die Zahnarztpraxis verlässt - und sie bald wieder betritt. Und: Er ist die beste Mund-zu-Mund-Propaganda!

Kinder, besonders Vorschulkinder, sind von Natur aus einer zauberhaften Welt voller Wunder sehr nahe. Zauberkunststücke bringen sie zum Staunen und bündeln ihre ganze Aufmerksamkeit. Zaubern ermöglicht so
Konzentration und Entspannung zugleich - es ist ein wunderbares Medium, um unruhige Kinder zur Ruhe kommen zu lassen und ängstliche Kinder zu lockern. Aufmerksame, entspannte Kinder sind in der Lage, die erforderlichen Handlungsanweisungen zu verstehen und umzusetzen. Oft genügt schon ein einziges therapeutisches Zauberkunststück: Es verzaubert die ganze Atmosphäre, und die Zahnuntersuchung oder -behandlung kann nach kurzer Zeit erfolgreich durchgeführt werden (Abb. 1).

\section{Besuche und Überraschungen im Zauberzimmer}

Auch regelrecht panische Kinder können sich einlassen, wenn eine Überraschung und eine positive Umdeutung sie dann doch neugierig machen: Denn alles ist anders! Die Zahnarztpraxis hat sich in eine "Zauberpraxis» verwandelt. Und das Kind soll nun im «Zauberzimmer» einfach nur darauf achten, dass sein Mund zubleibt und die Zahnzauberin ihm dieses Mal all die Verwandlungen zeigt.

So können sich die kleinen Patienten erst einmal an den «Zauberstuhl»

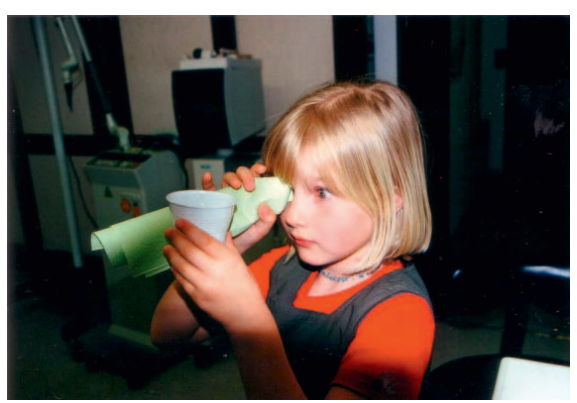

Abb. 1. Therapeutische Zauberkunststücke begleitend zur Zahnbehandlung. und an den "Zahnzauberer» gewöhnen (Abb. 2) und ein Zauberkunststück mit einer Zaubergeschichte erleben, die die ängstigende Situation annimmt und eine Lösung aufzeigt. So entstehen Mut und neue Selbstsicherheit.

Das therapeutische Zauberkunststück «Mutzauber» wird mit einer Mut machenden Geschichte präsentiert. Das lange, Nerven zehrende Bemühen, Warten und Flehen, bis das Kind «endlich den Mund aufmacht», entfällt so, Behandelte und Behandelnde erleben Spielfreude und gute Gemeinsamkeit. Und noch mehr: Sie können etwas, was eigentlich nicht möglich ist! Das gibt Selbstvertrauen, denn die Kunststücke des Therapeutischen Zauberns ${ }^{\circledR}$ leben von der Mitarbeit der Patienten: Ihre Zauberpuste, ihre Konzentration ist entscheidend für das Gelingen eines Zauberkunststücks.

Jedoch nicht nur Kinder, sondern auch erwachsene Patienten und sogar Jugendliche mit Null-Bock-Haltung sprechen auf individuelle Zauberkunststücke mit therapeutischen Geschichten an. Diese Geschichten beziehen Elemente aus der Hypnotherapie ein und können so tiefgehend

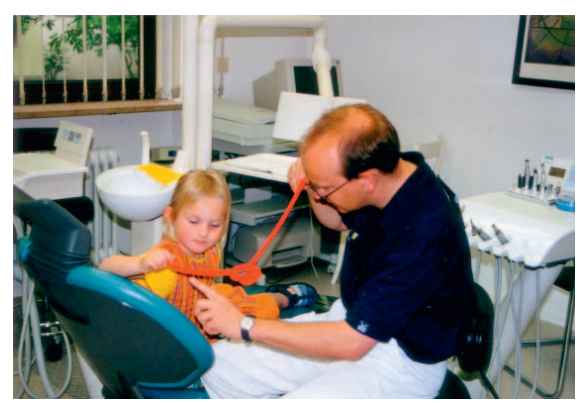

Abb. 2. Die «zauberhafte» Zahnarztpraxis: Zauberstuhl und Zahnzauberer. (c) 2011 S. Karger GmbH, Freiburg
Annalisa Neumeyer

Institut für Therapeutisches Zaubern ${ }^{\circledR}$

Eichrodtstrasse 13, 79117 Freiburg, Deutschland

Tel. +49761 61290972

neumeyer@therapeutisches-zaubern.de

www.therapeutisches-zaubern.de 
beruhigen und entspannen. Die Ablenkung auf angenehme Körperzustände oder Imaginationen erleichtert unangenehme Untersuchungen oder Behandlungen.

Setzen Sie Jugendlichen und Erwachsenen also einfach die rosarote Zauberbrille auf und schicken Sie sie auf eine Zauberreise zu ihren persönlichen guten Erinnerungen und Erfolgen!

\section{Ängstliche Eltern aus dem Behandlungszimmer zaubern}

Viele Eltern, die mit ihren Kindern in die Zahnarztpraxis kommen, stärken ihre Kinder, indem sie Sicherheit und Zuversicht vermitteln. Manchmal haben Eltern allerdings mit eigenen Ängsten aus der Kindheit zu kämpfen. Diese Ängste sitzen dann - meist unbewusst und kaum steuerbar - mit im Behandlungszimmer und werden für alle zum Hindernis. Dann ist es besser, wenn der Zahnzauberer die Mutter oder den Vater in das Wartezimmer zaubert.

Die Eltern sollen es sich im Wartezimmer gut gehen lassen, während das Kind im Zauberzimmer zum «stärksten Kind der Welt» gemacht wird. Wie jedoch ist das grosse Zaubergeheimnis, und deshalb müssen die Eltern draussen warten.

Fast alle Eltern spielen da gerne mit. Und die kleinen Patienten erleben eine ganz besondere Motivation zur Mitarbeit: Der Zahnzauberer führt mit ihnen den «Kraftzauber» durch. Später kann das Kind diese Kraft den Eltern im Wartezimmer und später auch allen zu Hause oder in der Schule zeigen.

\section{Zauberworte}

Besonders in schwierigen Situationen ist Sprache von grosser Bedeutung. Worte können beruhigende, stärkende und ermutigende Wirkung haben.
Solche «Zauberworte» sind:

- Positiv ausgedrückte, klare Handlungsanweisungen: Sagen Sie statt «Du brauchst keine Angst zu haben!» besser: "Du darfst es Dir bequem machen, und wir helfen Dir, Deinen Körper zu lockern.» Und statt «Das war doch gar nicht so schlimm!» besser: «Das ist doch richtig gut gegangen!» Denn NichtSätze bedeuten, dem Kind zu sagen, was es nicht tun soll, jedoch offen zu lassen, was es soll, und gleichzeitig zu erwarten, dass das Kind das tut, was wir uns vorstellen. Nehmen Sie Ihre Sprache einmal unter die Lupe und sammeln Sie eine Woche lang alle Negativ-Formulierungen mit dem Ziel, sie positiv neu zu formulieren. Viel Spass dabei!

- Formulierungen, die den Patienten anerkennen: Beispielsweise könnten Sie statt «Da hast Du aber nicht gut geputzt» sagen: «Ich weiss, manchmal hat man keine Lust zum Zähne bürsten. Ich zeige Dir, wie es ganz genau geht, und dann bitten wir noch Deine Mama, Dich zu erinnern.»

- Konsequente Fokussierungen auf positiv Bestehendes und auf Ziele: So zeigt die Zahnzauberin dem Kind, welche Zähne besonders gut geputzt und gesund sind. Vielleicht könnte es den anderen Zähnen auch diese wunderbare Pflege gönnen.

- Positive Umdeutungen und Umbenennungen: Putzen finden die meisten Menschen langweilig. Warum also sollte ein Kind Spass am Zähne putzen haben? Die Zähne mit der Bürste kitzeln oder kleine, weisse Wolken auf die Zähne malen, ist dagegen viel schöner.

\section{Die Gruppenprophylaxe verzaubern und bereichern}

Therapeutisches Zaubern kann spontan und spielerisch auch bei Pro- phylaxeveranstaltungen in Kindergärten und Schulen eingesetzt werden. Dabei geht es in erster Linie darum, Kinder am magischen Geschehen teilhaben zu lassen, also um ein gemeinsames Tun und Erleben. Als «Zauberkinder» übernehmen die zukünftigen kleinen Patienten gerne eine aktive Rolle. So wird nicht nur ein positiver Kontakt aufgebaut, sondern die Kinder können ihre Erfahrungen beim gemeinsamen Zaubern zudem übertragen: Ebenso wie beim Zaubern ist ihre Mitarbeit auch bei der Untersuchung, Behandlung und Pflege ihrer Zähne notwendig und wertvoll.

\section{Eine zauberhafte \\ Zahnarztpraxis entsteht}

Wenn Sie Interesse an einem positiveren und zauberhaften Umgang mit Ihren Patientinnen und Patienten bekommen haben und sich darüber freuen, wenn Gedanken an Ihre Zahnarztpraxis immer positive Erinnerungen und ein inneres Lächeln wachrufen, können Sie bei der Autorin Zauberfortbildungen besuchen. Mit dem Zaubergeist im Haus arbeitet es sich leichter!

Nach Absprache sind auch Schulungen einzeln oder im Team möglich. Unter www.Therapeutisches-Zaubern. de finden Sie Informationen über Rahmenbedingungen, Inhalte und aktuelle Termine der Zauberfortbildungen im Internet.

\section{Literatur}

Neumeyer A: Zaubern in der Zahnarztpraxis mit magischen Tricks und Zauberworten die Angst vor dem Zahnarzt wegzaubern; in Maiwald H-J (Hrsg): Kinderzahnheilkunde. Aktualisierungsauslieferung. Balingen, Spitta, 2000 .

Neumeyer A: Fall nicht! Warum Kinder vom Klettergerüst fallen, obwohl wir ihnen immer wieder sagen, dass sie nicht herunterfallen sollen. Ein Artikel über positive und negative Sprache bei Kindern. DHZ Heft 5/2000.

Neumeyer A: Mit Feengeist und Zauberpuste, ed 4. Freiburg, Lambertus, 2009.

Neumeyer A: Wie Zaubern Kindern hilft, ed 2. Stuttgart, Klett-Cotta, 2009. 